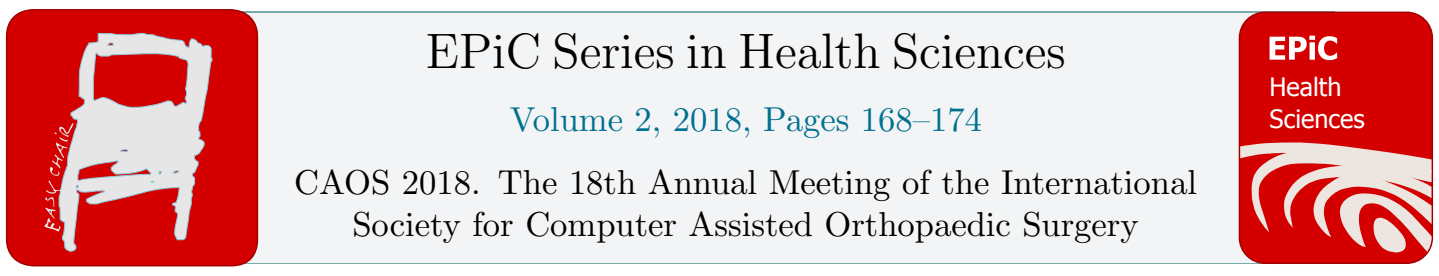

\title{
Automatic Scan Plane Identification from 2D Ultrasound for Pedicle Screw Guidance
}

\author{
Xiao Qi ${ }^{1 *}$, Nilay Vora ${ }^{2}$, Luis Riera ${ }^{2}$, Amrut Sarangi ${ }^{2}$, \\ George Youssef ${ }^{2}$, Michael Vives ${ }^{3}$, and Ilker Hacihaliloglu ${ }^{2,4}$ \\ ${ }^{1}$ Department of Electrical and Computer Engineering, Rutgers University, USA \\ ${ }^{2}$ Department of Biomedical Engineering, Rutgers University, USA \\ ${ }^{3}$ Department of Orthopaedics, Rutgers New Jersey Medical School, USA \\ ${ }^{4}$ Department of Radiology, Rutgers Robert Wood Johnson Medical School, USA \\ xiao.qi@rutgers.edu ilker.hacesoe.rutgers.edu
}

\begin{abstract}
In order to reduce the total amount of radiation exposure and provide real-time guidance ultrasound has been incorporated as a potential intra-operative imaging modality into various orthopedic procedures. However, high levels of noise, various imaging artifacts, and bone boundaries appearing several millimeters in thickness hinder the success of ultrasound as an alternative imaging modality in assisting orthopedic surgery procedures. Additional difficulties are also encountered during manual operation of the ultrasound transducer during image acquisition. In this work, we proposed a combination of novel scan plane identification method, based on convolutional neural networks, and bone surface localization method. The bone surface localization approach utilizes both local phase information, a combination of three different local image phase information and signal transmission map obtained from an L1 norm based contextual regularization method. The proposed network was utilized on two different US systems and to identify five different scan planes. Validation was performed on scans obtained from 16 volunteers. The correct scan plane identification rate of over $93 \%$ has been obtained. Validation against expert segmentation achieved a mean vertebra surface localization error of $0.42 \mathrm{~mm}$.
\end{abstract}

\section{Introduction}

Spinal fusion surgeries increased by $113 \%$ between the years 1998 - 2011 (The Burden of Musculoskeletal Diseases in the United States (BMUS), 2014). Treatment involves insertion of pedicle

\footnotetext{
${ }^{*}$ Corresponding author
} 
screws. Due to the close proximity of many important neural and vascular structures, surrounding the surgical area accurate screw placement is important (Manbachi, et al., 2014). Accuracy of screw placement impacts fusion rate and it also impacts adjacent level disease. Percutaneous pedicle screw insertion (PPSI) procedures have gained widespread acceptance due to advantages such as reduced length of stay, reduced blood loss and earlier return to work (Mobbs, et al., 2011). Computer assisted orthopedic surgery (CAOS) systems have increased the success rates of PPSI procedures (Gelalis, et al., 2012). However, the clinical accuracy requirements for certain levels of spine still exceed the accuracy of current image guided surgical systems. Another major concern is the exposure to ionizing radiation since most of the currently available PPSI systems are based on two-dimensional (2D)/ threedimensional (3D) intra-operative fluoroscopy.
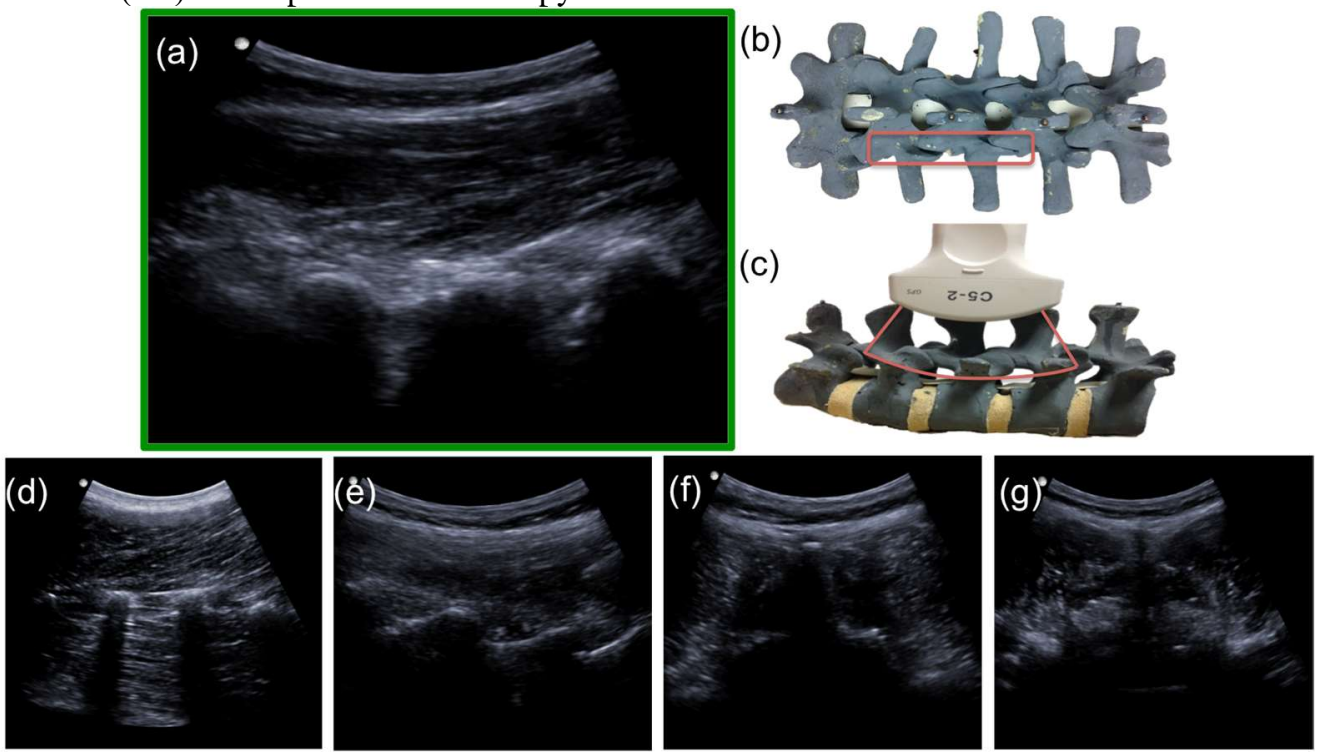

Figure 1: Five different types of scan planes of lumbar spine. (a) Paramedian sagittal articular process view corresponding to the scan plane overlapping the pedicle region (correct scan plane). (b-c) Schematic figure showing the US transducer orientation for correct scan-plane. (d) Paramedian sagittal transverse process view. (e) Paramedian sagittal laminar view. (f) Transverse spinous process view. (g) Transverse interlaminar view.

Ultrasound (US) has been incorporated into various CAOS procedures due to its real-time, portable, less expensive, non-radiation, and 3D imaging capabilities (Dardenne, et al., 2009). Compared to standard fluoroscopy imaging, US bone images have low signal to noise ratio (SNR), various imaging artifacts which do not correspond to any anatomical structure, and bone surfaces appear several millimeters in thickness. Another important limitation of US is the need for manual operation of the transducer which effects the quality of the collected bone data. Correct localization of the US transducer with respect to the bone anatomy is especially important while imaging complex anatomy bone surfaces such as the spine. In order to overcome some of these limitations several research groups have proposed methods for automatic extraction of bone surfaces from the collected intra-operative US data (Anas, et al., 2015; Beitzel, et al., 2012; Hacihaliloglu, et al., 2015; Kowal, et al., 2007; Quader, et al., 2014). However, a combination of automatic scan plane identification and bone segmentation has not been investigated previously.

With these in mind, we propose a novel pedicle screw fixation scan plane selection method, based on convolutional neural networks (CNNs), from 2D US data. The proposed system can utilize the proposed $\mathrm{CNN}$ to guide surgeon to the correct scan plane during the procedure, and thus overcome the 
disadvantage of US images not corresponding to any anatomical structure. During the final stage of our proposed method, the bone surfaces are extracted using a local phase-based bone segmentation method (Hacihaliloglu, 2018). Eventually, the local phase based enhancement approach highlights the spine surface, which could be incorporated into spine model registration and segmentation (Hacihaliloglu, et al., 2014), which could provide a better view when screws go under the bone surface. We validate the proposed method on 2D US scans collected from 16 volunteers. Here, this work is presenting in preliminary form testing only in one sagittal AP plane, and future proposed system will provide both sagittal and transvers plan in assisting surgeon to more accurately place pedicle screws.

\section{Materials and Methods}

\subsection{Data Collection}

434 US lumbar spine images were captured from 10 subjects by a Sonix-Touch US system (Ultrasonix, Medical Crop, Richmond, BC, Canada); it includes a collection of images from five different scan planes shown in Figure 1. In addition, 104 US lumbar spine images were collected from 6 subjects including three different scan planes by a wireless portable US scanner (Clarius C3, Clarius Mobile Health, Burnaby, BC, Canada) in order to increase size of database and test accuracy of proposed network on different US system. In vivo volunteer scans were collected after obtaining the approval of Rutgers University institutional review board (IRB). The detailed information regarding number of images in each scan plane for two models of US systems are summarized in Table 1. All the collected scans were cropped into a 512x512 region of interest (ROI), which includes only the vertebrae bone surfaces (Fig. 1.). The articular process (AP) scan plane, during longitudinal paramedian sagittal scanning approach, covers part of pars interarticularis and the inferior point of the superior articular facet. Therefore, AP scan plane is a proper scan plane as reference for placement of pedicle screw from sagittal view.

\begin{tabular}{|c|c|c|}
\hline View & $\begin{array}{c}\text { Sonix-Touch US } \\
\text { System }\end{array}$ & $\begin{array}{l}\text { Wireless Portable } \\
\text { US Scanner }\end{array}$ \\
\hline $\begin{array}{l}\text { Paramedian Sagittal Articular } \\
\text { Process View }\end{array}$ & 130 & 69 \\
\hline $\begin{array}{l}\text { Parameidan Sagittal Transvers } \\
\text { Process View }\end{array}$ & 91 & 21 \\
\hline $\begin{array}{l}\text { Paramedian Sagittal Laminar } \\
\text { View }\end{array}$ & 92 & 16 \\
\hline Transver Spinous View & 59 & $\mathrm{~N} / \mathrm{A}$ \\
\hline Transver Interlaminar View & 62 & N/A \\
\hline $\begin{array}{c}\text { Total: } \\
\end{array}$ & 434 & 106 \\
\hline
\end{tabular}

Table 1: Number of Images for Each Type Collected by Different US Systems 


\subsection{Automatic Scan Plane Selection using CNNs}

The proposed network architecture is inspired by the AlexNet, which consists of 5 convolutional layers and 3 fully-connected layers (Krizhevsky, et al., 2012). In our work, we introduce some of changes to the original network in order to optimize the CNN to obtain prediction for larger $512 \times 512$ input images, and this architecture would make a prediction according to class score of each category.

We trained the network using mini-batch stochastic gradient descent (SGD) with a momentum of 0.9 , a cross-entropy loss, and with a learning rate of 0.001 for both Sonix-Touch US system and wireless portable US scanner. In order to reduce overfitting and make the network more robust to identification AP scan planes, we performed data augmentation which resulted in the generation of additional datasets which are used to train our network. Data augmentation is achieved using horizontal flip, and introduced four different translations. By performing this data augmentation, we enlarged our data set ten times resulting in the training data size of 3840 and 560 for the Sonix-Touch and Wireless US scanners respectively. Training for both US systems was performed using the random sampling method where 50 images were randomly selected from AP scan planes dataset for testing and the rest AP scan plane images and all other US images from the rest four scan planes were used for training after augmentation (Bishop, 1995). To further solidify the identification accuracy, we repeated the process of random selection of AP scan plane image five times and averaged the identification accuracy over these five times testing (5-fold cross validation).

\subsection{Bone Segmentation}

The proposed bone segmentation method is based on the extraction of three different image phase features using band-pass quadrature filters (Hacihaliloglu, 2017). The extracted phase features are used as an input to an $\mathrm{L}_{1}$ norm-based contextual regularization method for the enhancement of bone shadow regions. Recently this method was also applied for the enhancement of bone shadow regions corresponding to extended field of view spine US data (Alsinan, et al., 2017). In this work, in order to extract bone surface features from the automatically localized scan planes, we combine local phase image features and the enhanced bone shadow regions into a dynamic programming solution (Hacihaliloglu, 2018) The dynamic programming method segments a bone surface for each scanline in the B-mode US data. A bone probability image, obtained by masking the enhanced shadow image with the local phase images, is used to guide the optimization. The dynamic programming approach minimizes a cost function in order to calculate a path, denoted as $B L(s)$, corresponding to the bone surfaces present in the US data. During this optimization US image partitioned into three different regions denoted as bone, boneless, jump (Fig.2-d). The proposed bone segmentation method is validated on 89 vertebrae surfaces obtained from 16 volunteer scans. Gold standard surfaces are obtained by manual expert segmentation. The quality of the segmentation is evaluated by computing average Euclidean distance (AED) between the manual and automatically extracted bone surfaces. 


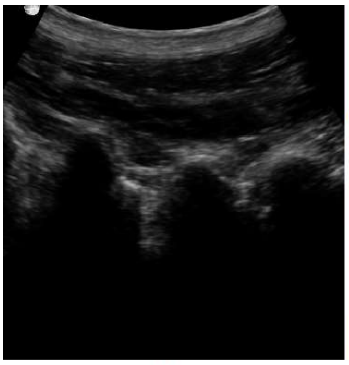

(a)

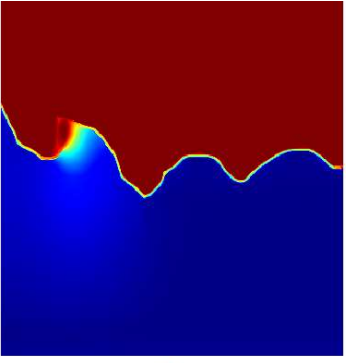

(b)

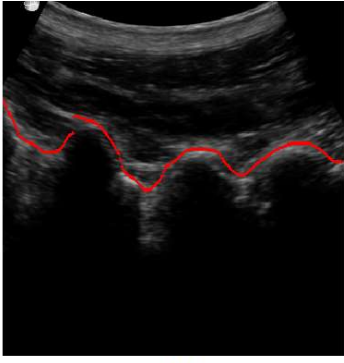

(c)

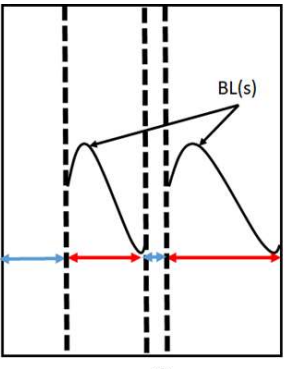

(d)

Figure 2: (a) Paramedian sagittal AP view of lumbar spine B-mode US image identified from the proposed CNN method. (b) Bone shadow enhanced image. (c) Bone localization result. Automatically identified bone surfaces are displayed in red color. (d) Schematic plot of US regions used during dynamic programming-based bone segmentation. Blue arrows correspond to boneless regions, red arrows correspond to bone regions, and black vertical dashed bars correspond to jump regions.

\section{Results}

\subsection{AP Scan Plane Identification}

The average identification accuracy with maximal and minimal values for both US systems are presented in Table 2. From Table 2, it can be seen that the proposed network has equivalently high accuracy for images collected from both US systems. 11 images in 17 misidentified images were considered as laminar scan plane by the proposed network for Sonix-Touch system. Similarly, 10 of 16 misidentified images from wireless portable system were misclassified as laminar scan plane. The training time using the dataset obtained from the Sonix-Touch US system was 7.5 hours. Training time decreased to 1.09 hours for the Wireless US system due to less number of images being used. Classification time for a single 2D US scan was 0.09 seconds.

\begin{tabular}{lrr}
\hline US system & $\begin{array}{r}\text { Averaged } \\
\text { Accuracy }\end{array}$ & $\begin{array}{r}\text { Min. } \\
\text { Accuracy }\end{array}$ \\
\hline Sonix-Touch & $93.2 \%$ & $90 \%$ \\
Wireless Portable & $93.6 \%$ & $88 \%$ \\
\hline Table 2: AP Scan Plane Identification Accuracy
\end{tabular}

\subsection{Bone Segmentation}

Mean AED error for localization of vertebra bone surfaces was $0.43 \mathrm{~mm}$ (SD:0.19 mm). The maximum AED error was $1.15 \mathrm{~mm}$. The combination of enhanced local phase bone features and shadow region information provides a robust estimate for the spine features from the extracted scan plane. Qualitative result from the bone segmentation is presented in Figure 3. Computaiton time for a single scan was 9.4 seconds using an unoptimized MATLAB code. 


\section{Discussion and Future Work}

Most of misidentification happened between AP scan plane and laminar scan plane. This is due to laminar is adjacent to AP, and thus when probe slightly tilts, it would cause AP scan plane US images have a high similarity with laminar scan plane images. In addition, two images from Sonix-Touch system were considered as interlaminar scan plane image by the proposed network. For minimization of mentioned error and enhancement of classification accuracy, the network should be trained by a large amount of US images under different views. Moreover, the application of preprocessing techniques is able to provide more distinguished features to neutral network and could further improve accuracy.

Generally, the optimal entry point of pedicle screws is at the intersection of the pars interarticularis, the midpoint of the transverse process, and the inferior point of the superior articular facet (Koon, 2010; Oh, et al., 2013). Using US as a type of guidance would potentially provide a real-time imaging alternative for the identification of dorsal anatomic landmarks. The proposed $\mathrm{CNN}$ classifier provides a good reference for surgeon in identification of proper scan planes. The reported time of 9.4 seconds for segmentation of bone surfaces needs to be improved in order for the proposed classification and segmentation method to be used for intra-operative guidance.

Future work will investigate the ability of CNNs in identification of transvers plane of different level of lumbar spine. It is important that each surgeon may require to review in both sagittal and transverse planes for accurate placement of pedicle screws. We will also investigate incorporation of data from more patients to increase the size of training data set in order to improve classification accuracy. Finally, investigation of CNNs for segmentation of the bone surfaces from the US data will also be performed as part of our future work.

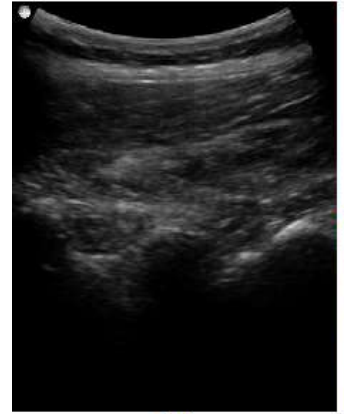

(a)

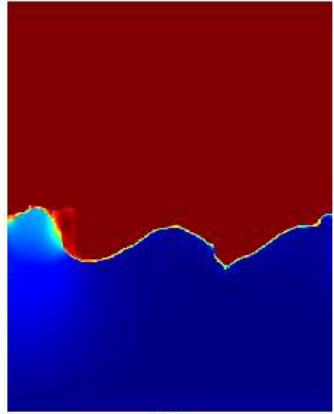

(b)

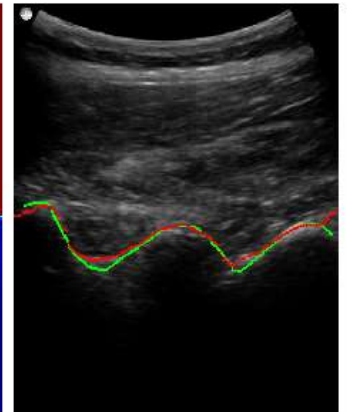

(c)

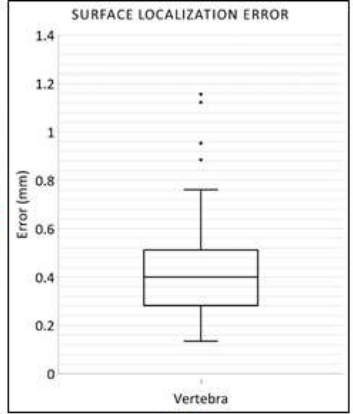

(d)

Figure 3: (a) Paramedian sagittal AP view of lumbar spine B-mode US image identified from the proposed CNN method. (b) Bone shadow enhanced image. (c) Bone localization result. Automatically identified bone surfaces are color coded in red, and manual expert bone segmentation is color coded in green. (d) Box and whisker plot for vertebra surface localization.

\section{Acknowledgement}

This work was supported by the North American Spine Society 2017 Young Investigator Basic Research Grant. 


\section{References}

Alsinan, Z. et al., 2017. Ultrasound transmission maps for enhancement of spine bone shadow region from extended field of view ultrasound data. Bone Joint Journal, 1 12.pp. pp. 39-43.

Anas, E. M. et al., 2015. Bone enhancement in ultrasound using local spectrum variations for guiding percutaneous scaphoid fracture fixation procedures. International journal of computer assisted radiology and surgery, 10(6), pp. pp. 959-69.

Beitzel, J. et al., 2012. Ultrasound bone detection using patient-specific ct prior. s.l., s.n., pp. pp. 2664-2667.

Bishop, B. C., 1995. Neural Networks for Pattern Recognition. s.1.:Oxford University Press.

Dardenne, G. et al., 2009. Toward a dynamic approach of THA planning based on ultrasound. Clinical orthopaedics and related research, pp. pp. 901-8.

Gelalis, I. D. et al., 2012. Accuracy of pedicle screw placement: a systematic review of prospective in vivo studies comparing free hand, fluoroscopy guidance and navigation techniques. European Spine Jounarl, 21(2), pp. pp. 247-255.

Hacihaliloglu, I., 2017. Enhancement of bone shadow region using local phase-based ultrasound transmission maps. International journal of computer assisted radiology and surgery, June, 12((6)), pp. pp. 951-60.

Hacihaliloglu, I., 2018. Localization of Bone Surfaces from Ultrasound Data Using Local Phase Information and Signal Transmission Maps. Computational Methods and Clinical Applications in Musculoskeletal Imaging, pp. pp 1-11.

Hacihaliloglu, I., Guy, P., Hodgson, A. J. \& Abugharbieh, R., 2015. Automatic extraction of bone surfaces from 3D ultrasound images in orthopaedic trauma cases. International journal of computer assisted radiology and surgery, 10((8)), pp. pp.1279-87.

Hacihaliloglu, I., Rasoulian, A., Abolmaesumi, P. \& Rohling, R. N., 2014. Local Phase Tensor Features for 3D Ultrasound to Statistical Shape+Pose Spine Model Registration. IEEE Transaction on Medical Imaging (TMI) , 33(11), pp. pp. 2167 - 2179.

Hacihaliloglu, I., Rasoulian, A., Abolmaesumi, P. \& Rohling, R. N., 2014. IEEE Transaction on Medical Imaging (TMI). Local Phase Tensor Features for 3D Ultrasound to Statistical Shape+Pose Spine Model Registration, 33(11), pp. pp. 2167-2179.

Koon, J., 2010. In: Surgical atlas of spine. 1 ed. s.1.:Korean Spinal Neurosurgery Society, pp. pp. 267-276.

Kowal, J. et al., 2007. Automated bone contour detection in ultrasound B-mode images for minimally invasive registration in computer-assisted surgery: An in vitro evaluation. The International Journal of Medical Robotics and Computer Assisted Surgery, 3((4)), p. pp. 341-348.

Krizhevsky, A., Sutskever, I. \& Hinton, G. E., 2012. ImageNet Classification with Deep Convolutional Neural Networks. Neural Information Processing Systems, pp. pp. 1097-1105.

Manbachi, A., Cobbold, R. S. \& Ginsberg, H. J., 2014. Guided pedicle screw insertion: techniques and training. The Spine Journal, 14(1), pp. pp. 165-179.

Mobbs, R. J., Sivabalan, P. \& Li, J., 2011. Technique, challenges and indications for percutaneous pedicle screw fixation. Journal of Clinical Neuroscience, 18(6), pp. pp.741-749.

Oh, C. H. et al., 2013. Technical Report of Free Hand Pedicle Screw Placement using the Entry Points with Junction of Proximal Edge of Transverse Process and Lamina in Lumbar Spine: Analysis of 2601 Consecutive Screws. Korean J Spine, 10(1), pp. pp. 7-13.

Quader, N., Hodgson, A. \& Abugharbieh, R., 2014. Confidence weighted local phase features for robust bone surface segmentation in ultrasound. s.1., s.n., pp. pp. 76-83.

The Burden of Musculoskeletal Diseases in the United States (BMUS), 2014. United States Bone and Joint Initiative. [Online]. Available at: http://www.boneandjointburden.org/[Accessed 2582017 ]. 